\title{
Does Anticholinergic Activity Affect Neuropathology? Implication of Neuroinflammation in Alzheimer's Disease
}

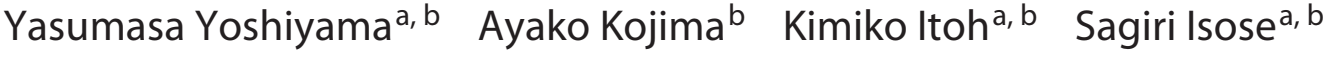 \\ Mizuho Koide ${ }^{a}$ b Koji Horic Kimihito Arai ${ }^{\mathrm{a}}$ \\ ${ }^{a}$ Department of Neurology and ${ }^{b}$ Clinical Research Center, Chiba-East National Hospital, Chiba, and ${ }^{\mathrm{C}}$ Department of \\ Psychiatry, Showa University Northern Yokohama Hospital, Yokohama, Japan
}

\section{Key Words}

Anticholinergic activity $\cdot$ Neuropathology $\cdot$ Alzheimer's

disease $\cdot$ Tau pathology $\cdot$ Neuroinflammation

\begin{abstract}
One characteristic neuropathological feature of Alzheimer's disease (AD) is profound neuronal loss in the nucleus basalis of Meynert, the major source of cholinergic innervation of the cerebral cortex. Clinically, anticholinergic activity causes a decline in cognitive function and increases the risk of dementia, thus possibly enhancing AD pathologies and neurodegeneration. Until now there has been insufficient human neuropathological data to support this conclusion. Experimental studies using a tauopathy mouse model demonstrated anticholinergics enhanced tau pathology and neurodegeneration corresponding to central anticholinergic activity. Additionally, donepezil, a cholinesterase inhibitor, ameliorated tau pathology and neurodegeneration in the same mouse model. These results indicate the balance between cholinergic and anticholinergic activities might affect neurodegeneration. Importantly, neurodegeneration observed in the mouse model seemed to correspond to the distribution of microglial activation, and it was reported that neuroin-
\end{abstract}

flammation plays an important role in the pathomechanism of $A D$, while anticholinergic activity augments inflammatory responses. Moreover, some studies indicated $\beta$-amyloid itself depletes cholinergic function similarly to anticholinergic activity. Thus, anticholinergic activity might initiate and/or accelerate $A D$ pathology. Limited human data support the conclusion that anticholinergic activity enhances AD-related neuropathology and neurodegeneration. However, experimental data from a tauopathy mouse model indicated anticholinergic activity might enhance neurodegeneration with enhanced neuroinflammation including microglial activation.

(c) 2015 S. Karger AG, Basel

\section{Introduction}

Cholinergic function, which plays an important role in learning and memory, might be regulated by the balance of cholinergic activity and anticholinergic activity. It is well known that central cholinergic activity becomes profoundly depleted, with severe neuronal loss in the nucleus basalis of Meynert, the major source of cholinergic innervation of the cerebral cortex, in Alzheimer's disease (AD)

\section{KARGER 125}

(c) 2015 S. Karger AG, Base

$1660-2854 / 15 / 0153-0140 \$ 39.50 / 0$

E-Mail karger@karger.com

www.karger.com/ndd
Yasumasa Yoshiyama

Department of Neurology and Clinical Research Center

Chiba-East National Hospital, 673 Nitona, Chuo-ku

Chiba, Chiba 260 (Japan)

E-Mail neuroyy@cehpnet.com 
[1]. Additionally, substantial neocortical deficits in the enzyme responsible for the synthesis of acetylcholine (ACh), choline uptake and ACh release are reduced [2]. These observations led to the 'cholinergic hypothesis of $A D$ ', wherein degeneration of cholinergic neurons in the basal forebrain and the associated loss of cholinergic neurotransmission are major contributing factors to the deterioration in cognitive function in $\mathrm{AD}$. However, the cholinergic hypothesis is not presently assumed as a primary etiology, but rather the consequence of neurodegeneration induced by the so-called 'amyloid hypothesis', comprised of the multiple pathological steps initiated by $\beta$-amyloid $(A \beta)$ abnormality leading to $A \beta$ oligomerization, abnormal tau aggregation, synaptic dysfunction, cell death and brain shrinkage in $\mathrm{AD}$. To compensate for cholinergic dysfunction in the brain, acetylcholinesterase inhibitors (AChEIs) have been widely used for treating $\mathrm{AD}$. There is increasing evidence of improved or stabilized cognitive decline, as well as behavioral and psychological symptoms with administration of AChEIs. Although the symptomatic efficacy of AChEIs is assumed to take place through augmentation of ACh-mediated neurotransmission, some studies suggest AChEIs might slow disease progression by suppressing brain atrophy [3-6]. On the other hand, anticholinergic drugs, particularly antimuscarinic drugs, which are widely used for treating overactive bladder or Parkinson's symptoms in elderly people, might induce cognitive decline and increased dementia symptom severity [7-11]. Moreover, many drugs commonly prescribed to elderly people have anticholinergic properties, for example antidepressants, antiemetics, antispasmodics, bronchodilators, antiarrhythmic drugs and antihistamines. A large-scale cohort study of elderly individuals identified mild cognitive impairment in $80 \%$ of patients under antimuscarinic therapies and concluded that this therapy imparts a fivefold increased risk of mild cognitive impairment [8]. There are significant associations reported between high serum anticholinergic activity and cognitive functions and psychiatric symptoms $[12,13]$. A recent systematic review indicated $77 \%$ of included studies evaluating cognitive function $(n=33)$ reported a significant decline in cognitive ability with increasing anticholinergic load $(\mathrm{p}<0.05)$ [14]. These observations gave rise to the presumption that anticholinergic activity might enhance $\mathrm{AD}$ pathologies and neurodegeneration. Here, we review the effect of anticholinergic activity on neuropathological changes and discuss the implications of neuroinflammation in those pathological changes.

Does Anticholinergic Activity Affect Neuropathology?

\section{Anticholinergic Activity and Neuropathological Changes in Humans}

Even if the risk of dementia is increased in people who use anticholinergics, it is difficult to conclude that anticholinergics enhance AD pathology. Because anticholinergics may cause a functional decline in cognitive function, patients with anticholinergics might easily show dementia symptoms even with mild AD pathology. To determine the effect of anticholinergics on $\mathrm{AD}$ pathology, a large and long-standing prospective neuropathological study including nondemented subjects is required, as well as the need to aggregate the drug information about the duration and dosage of anticholinergics. So far, no such study has been conducted. Perry et al. [15] retrospectively compared senile plaque (SP) and neurofibrillary tangle (NFT) densities in nondemented Parkinson disease (PD) patients. They divided these patients into 3 groups: longterm (2-18 years), short-term ( $<2$ years) treatment with anticholinergics and no anticholinergic medications. Anticholinergics are frequently prescribed in PD patients for controlling PD symptoms, especially tremors. Additionally, drugs for treating depression, pollakiuria and gastrointestinal symptoms with anticholinergic properties are commonly prescribed as concomitant drugs in PD patients. Therefore, $\mathrm{PD}$ patients seem a likely group in which to examine the neuropathological effects of anticholinergics. Neuropathological analysis of PD patients on anticholinergic drugs demonstrated significant differences in cortical SP and NFT densities between long-term anticholinergic treatment, and short-term treatment or no anticholinergic treatment. SPs were more than twofold higher in long-term treatment compared with short-term treatment $(p=0.00005)$ or no treatment $(p=0.005)$. Additionally, NFT densities were also twofold higher in long-term compared with short-term treatment $(\mathrm{p}=0.05)$ or no treatment $(p=0.02)$. Meanwhile, there were no significant differences of SP and NFT densities between short-term treatment and no-treatment groups. These results suggest long-term anticholinergic treatments might accelerate $\mathrm{AD}$ pathology. However, there were some critical limitations in this study. First, this study was retrospective and excluded PD patients with dementia. Thus, it is unclear whether anticholinergics enhance SPs and NFTs to the levels seen in $\mathrm{AD}$ or accelerate $\mathrm{AD}$ pathology in demented patients. Second, the pathological changes observed in this study were generally very mild, and cases with high NFT and/or high SP densities were excluded even in the absence of recorded dementia. Third, semiquantification of NFT and SP was documented in just one cortex (most

Neurodegener Dis 2015;15:140-148 141 
frequently recorded in the frontal one). Fourth, it is difficult to exclude the possibility that the tremor-predominant type of PD patients, to whom anticholinergics are most frequently prescribed, might be likely to have more AD pathology. Lastly, the study did not investigate other accumulated protein pathologies including $\alpha$-synuclein. Therefore, it is difficult to say if there is sufficient evidence at present of the association between anticholinergic activity and neuropathology in humans.

\section{Anticholinergic Activity and Experimental Neuropathological Studies}

Experimental studies to evaluate the effects of anticholinergics on neuropathology in laboratory animals are also difficult to find, except for a study published by our group. However, a few studies have analyzed the effects of cholinergic agents on neuropathological changes using laboratory animals. Here, we re-analyzed the joint data from 2 studies from our group using a tauopathy mouse model (PS19), one administering 2 anticholinergic drugs [16] and the other administering a cholinergic drug [17]. These 2 studies were performed at the same time, but we previously analyzed these data separately.

PS19 mice exhibit synaptic loss and microglial activation beginning at 3 months of age, followed by abnormal tau accumulation starting at 5 months. Neuronal loss and brain atrophy can be observed at about 8 months [18]. We used a tau mouse model instead of an amyloid mouse model because tau pathology is more directly related to neurodegeneration and clinical symptoms. Multiple in-

Fig. 1. Exacerbation of tau pathology and neuronal loss by anticholinergic agents, and amelioration by a cholinergic agent. Brain sections of PS19 mice treated with the cholinergic agent DZ (a, e, i, $\mathbf{m})$, anticholinergic agent $\mathrm{TP}(\mathbf{b}, \mathbf{f}, \mathbf{j}, \mathbf{n})$ or anticholinergic agent $\mathrm{PP}$ (c, g, k, o), or nontreated control (nTC; $\mathbf{d}, \mathbf{h}, \mathbf{I}, \mathbf{p}$ ) were immunohistochemically stained with a phosphorylation-specific tau antibody, AT8 (a-h) and Nissl staining (i-p). AT8 staining in the CA1 $(\mathbf{a}-\mathbf{d})$ and the CA3 (e-h) regions shows the proportion of AT8positive pyramidal neurons is slightly increased in PP-treated (c, g) and clearly increased in TP-treated PS19 mice (b, f), compared with nTC PS19 mice (d, h). Conversely, the number of AT8-positive pyramidal neurons is decreased in DZ-treated PS19 mice (a, e). In low-magnification images of hippocampus stained by Nissl staining (i-I), severe neuronal loss occurs in the CA3 region in TPtreated PS19 mice (j, arrowheads) and moderate neuronal loss in PP-treated PS19 mice (k). Higher magnification reveals many atrophic pyknotic neurons in TP-treated and nTC PS19 mice (n, arrowheads), but not in DZ-treated PS19 mice (m). The alteration dependent imaging/autopsy studies document that neurodegeneration, brain atrophy and clinical symptoms might correlate well with tau pathology, but not with amyloid pathology $[19,20]$. In addition, recent experiments in vivo or in vitro demonstrate that tau pathology seems to transsynaptically spread along neuronal networks corresponding to the development of $\mathrm{AD}$ pathology and clinical symptoms $[20,21]$. To investigate the effects of anticholinergic and cholinergic activity on tau pathology and neurodegeneration, 2 anticholinergic agents and 1 cholinergic agent were administered to suppress or enhance, respectively, the cholinergic system in the tauopathy mice. The 2 anticholinergic agents were trihexyphenidyl (Artane $\left.{ }^{\mathrm{TM}}, \mathrm{TP}\right)$, which is preferentially used for the treatment of PD in Japan, and propiverine (BUP- $4^{\mathrm{TM}}$, $\mathrm{PP}$ ), which is used for the treatment of overactive bladder and has a lower central anticholinergic action [22-24]. The cholinergic agent used was donepezil (Arisept ${ }^{\mathrm{TM}}$, DZ). All drugs were administered to mice from the age of 2 to 10 months, namely before development of synaptic loss and microglial activation are thought to occur in the brain (see details of samples and methods $[16,17]$ ).

\section{The Effects of Anticholinergic or Cholinergic Activity on Tau Pathology and Neurodegeneration}

To compare tau pathology in each group, brain sections were stained with AT8, an antibody specific to phosphorylated tau (fig. 1a-h). Although the distribution of tau-positive neurons in each group was similar (hippocampus, amygdala, entorhinal cortex, brainstem and spinal cord), PS19 mice with TP showed a much stronger tau pathology in all areas mentioned above including the

in shape and Nissl stainability of neurons is also seen in nTC PS19 mice (p, arrowheads). $\mathbf{a}-\mathbf{h}, \mathbf{m}-\mathbf{p}$ Scale bars $=10 \mu \mathrm{m}$. i-I Scale bars $=100 \mu \mathrm{m}$. $\mathbf{q}$ To examine tau solubility in PS19 mice treated with DZ, TP or PP, $2 \mu$ g of reassembly buffer (RAB)-HS-extracted protein, $4 \mu \mathrm{g}$ of radioimmunoprecipitation assay (RIPA) bufferextracted protein and $5 \mu \mathrm{l}$ of formic acid (FA)-extracted samples from brains of 10-month-old DZ-, TP- or PP-treated PS19 mice, and nTC mice were immunoblotted with 17026, a tau-specific antibody. The insoluble tau protein levels are clearly different in the FA fractions, with stronger signals following TP or PP treatment and weaker signals in DZ-treated PS19 mice. Moreover, slowly migrating tau bands in the FA fractions (arrow) are clearly observed in TP-treated PS19 mice. $\mathbf{r}$ The signal intensities of tau protein in the FA fractions from TP- and PP-treated PS19 brains are significantly stronger than those from DZ-treated PS19 brains. Marker $=$ $1 \mathrm{~N} 4 \mathrm{R}$ recombinant tau protein; ${ }^{*} \mathrm{p}<0.05,{ }^{* *} \mathrm{p}<0.01$ (ANOVA with post hoc test).

(For figure see next page.) 

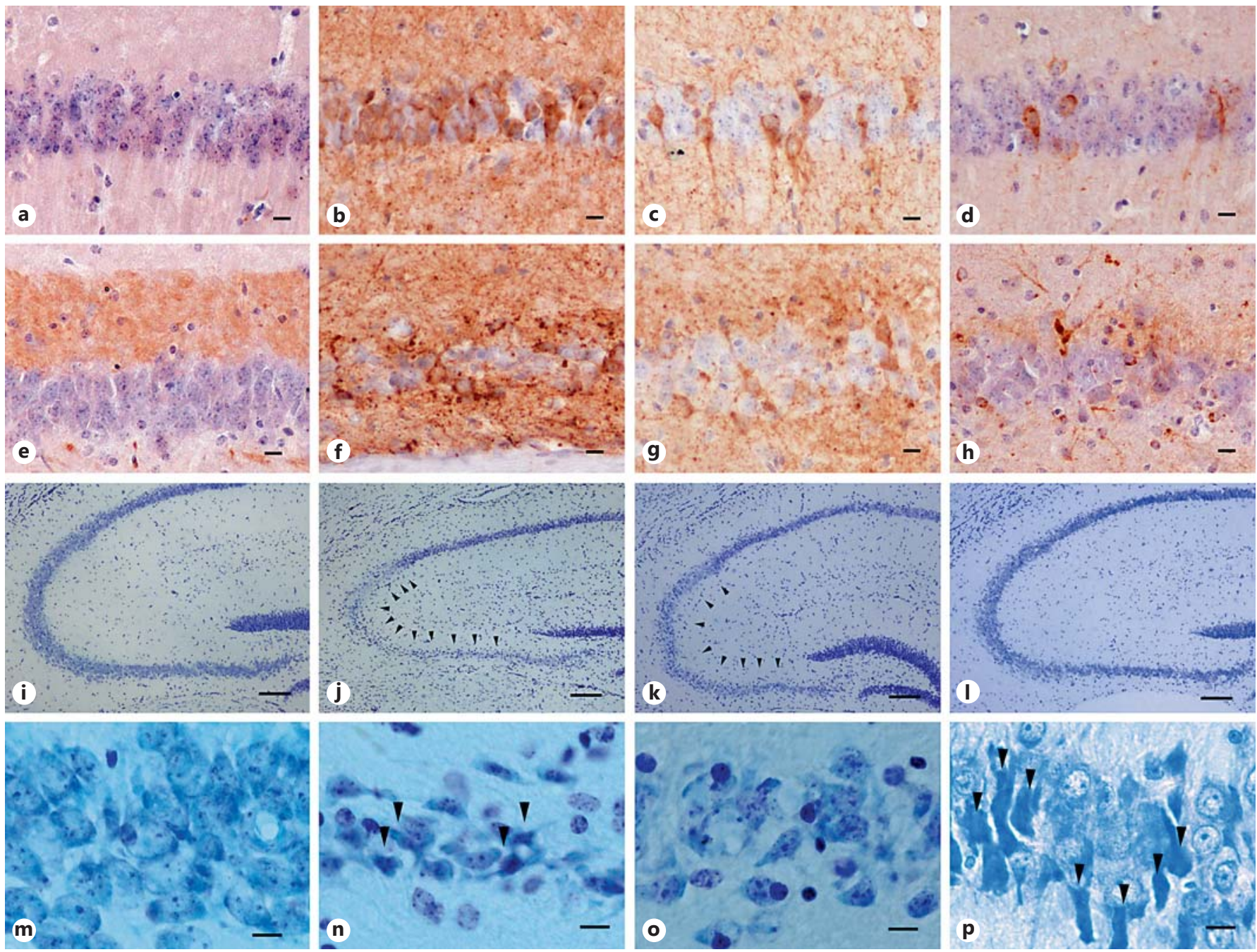

Tau DZ $\quad$ TP $\quad$ PP $\quad$ nTC

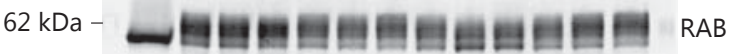

$47 \mathrm{kDa}$

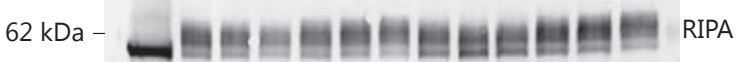

$47 \mathrm{kDa}-$

$62 \mathrm{kDa}-2$ 둘을

q

$47 \mathrm{kDa}-$

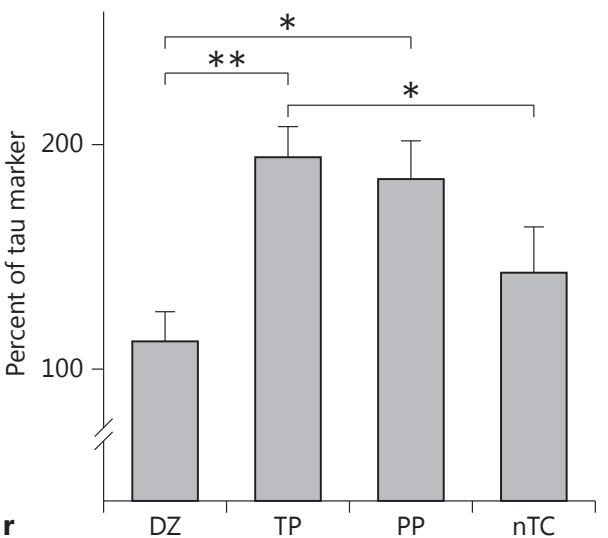


CA1 (fig. 1b) and CA3 (fig. 1f) regions, compared with nontreated control (nTC) PS19 mice (fig. 1d, h). Moreover, PP treatment caused an intermediate tau pathology phenotype between TP and nTC (fig. 1c, g). In contrast, PS19 mice administered DZ showed far fewer tau-positive neurons in the CA1 (fig. 1a) and CA3 (fig. 1b) regions. Because tau pathology is thought to be composed of insolubilized tau, tau insolubility was estimated by sequential extraction of brain samples with 3 buffers of increasing extraction strength - reassembly buffer $(\mathrm{RAB})<$ radioimmunoprecipitation assay $(\mathrm{RIPA})<$ formic acid (FA) - to estimate the solubility changes in tau [25]. Insoluble tau extracted with FA clearly demonstrated increased tau insolubility in TP and PP treatments. Conversely, FA samples demonstrated decreased tau insolubility in DZ (fig. 1q, FA). In particular, a slowly migrating band (fig. 1q, FA, arrow), which was highly phosphorylated, was more intense in TP-treated than in the PPtreated and nTC mouse brain samples in the FA fractions. Quantitative analysis of insoluble tau in the FA fractions indicated a significant increase in TP compared with nTC and DZ, and in PP compared with DZ (fig. 1r). Interestingly, signal intensities of soluble tau (RAB) in DZ seemed slightly stronger than in other treatments. This might be related to the reduction in insolubilized tau in RIPA and FA fractions (fig. 1q, r).

Next, to compare neurodegeneration, brain sections were stained by Nissl staining (fig. 1i-p). Low-magnification images of hippocampal regions (fig. 1i-l) revealed severe selective neuronal loss in the CA3 region, as well as hippocampal atrophy, in PS19 mice treated with TP and PP (fig. 1j, k, arrowheads, respectively) compared with nTC and DZ (fig. 11, i, respectively). Moreover, higher-magnification pictures (fig. $1 \mathrm{~m}-\mathrm{p}$ ) clearly displayed morphological changes in neurons, including pyknotic changes in nontreated (fig. 1p, arrowheads) and TP-treated PS19 mice (fig. 1n, arrowheads), as well as atrophic changes in TP-treated PS19 mice (fig. 1g). Interestingly, most atrophic or pyknotic neurons were not AT8-positive, suggesting the pathomechanism of neurodegeneration occurred without tau fibril accumulation. These data clearly demonstrated that anticholinergic activity accelerated tau pathology and neurodegeneration, and cholinergic activity showed the opposite effects.

The Effects of Anticholinergic or Cholinergic Activities on Microglial Activation

In PS19 mice, microglial activation preceded the development of tau pathology, and microglial activation was mainly observed in the hippocampus, amygdala, en- torhinal cortex and brainstem, corresponding to regions where tau pathology was dominantly observed, indicating the relationship between microglial activation, tau pathology and neurodegeneration. Moreover, early administration of an immunosuppressant, FK506, attenuated those pathologies in PS19 mice [18]. Therefore, microglia in the brains of DZ-, TP-, PP-treated and nTC PS19 mice were immunohistochemically analyzed with a microglial marker, Iba1. Double immunolabeling for Iba1 (red) and AT8 (green) clearly demonstrated distinctly suppressed microglial activation in the brains of DZ-treated PS19 mice (fig. 2d, parts $\mathrm{C} 1-\mathrm{C} 3$ ), whereas marked microglial activation occurred in the brains of TP-treated PS19 mice (fig. 2b, parts T1-T3, g, h) and moderate microglial activation occurred in the brains of PP-treated PS19 mice (fig. 2c, parts P1-P3) compared with nTC PS19 mice (fig. 2d, parts $\mathrm{C} 1-\mathrm{C} 3$ ). Higher-magnification images revealed enlarged, Iba1-positive microglia with thick processes in the CA3 region in TP-treated PS19 mice (fig. $2 \mathrm{~g}$ ), whereas a few, small microglia with thin processes (fig. 2e, inset) were observed in the nontransgenic wild-type mouse brain. In the CA1 region of TP-treated PS19 mice, microglia were elongated in parallel with the direction of neuronal processes (fig. $2 \mathrm{~h}$ ). Statistical analysis of the percentage of Iba1-positive areas in the CA1 or CA3 region clearly demonstrated that enhanced ACh levels caused by DZ treatment suppressed microgliosis in each hippocampal region, whereas suppression of the cholinergic system by TP or PP treatments enhanced microgliosis (fig. $2 \mathrm{i}, \mathrm{j}$ ). Interestingly, microglial activation might negatively depend on the central cholinergic activities $(\mathrm{DZ}>\mathrm{PP}>\mathrm{TP})$ modulated by cholinergic or anticholinergic activities.

Data mentioned above seem to indicate that anticholinergic and cholinergic drugs show opposite effects though the same mechanism. However, Western blot analysis of tau kinases demonstrated that DZ strongly suppressed JNK activity, but TP and PP did not enhance it $[16,17]$. An experiment using cell culture and adenoviral infection of tau demonstrated that tau overexpression in COS-7 cells induced JNK activation followed by excessive phosphorylation of tau, although JNK activation alone was insufficient to induce insoluble tau aggregation [26]. JNK is involved not only in tau phosphorylation, but also in the regulation of stress responses and the normal physiological processes of cell proliferation, apoptosis, differentiation and cell migration. Thus, suppression of JNK activity by DZ might relate not only to suppression of tau phosphorylation and aggregation, but also to neuronal survival. Additionally, glycogen synthase kinase $3 \beta$ and cyclin-dependent kinase 5, major tau kinases, were 

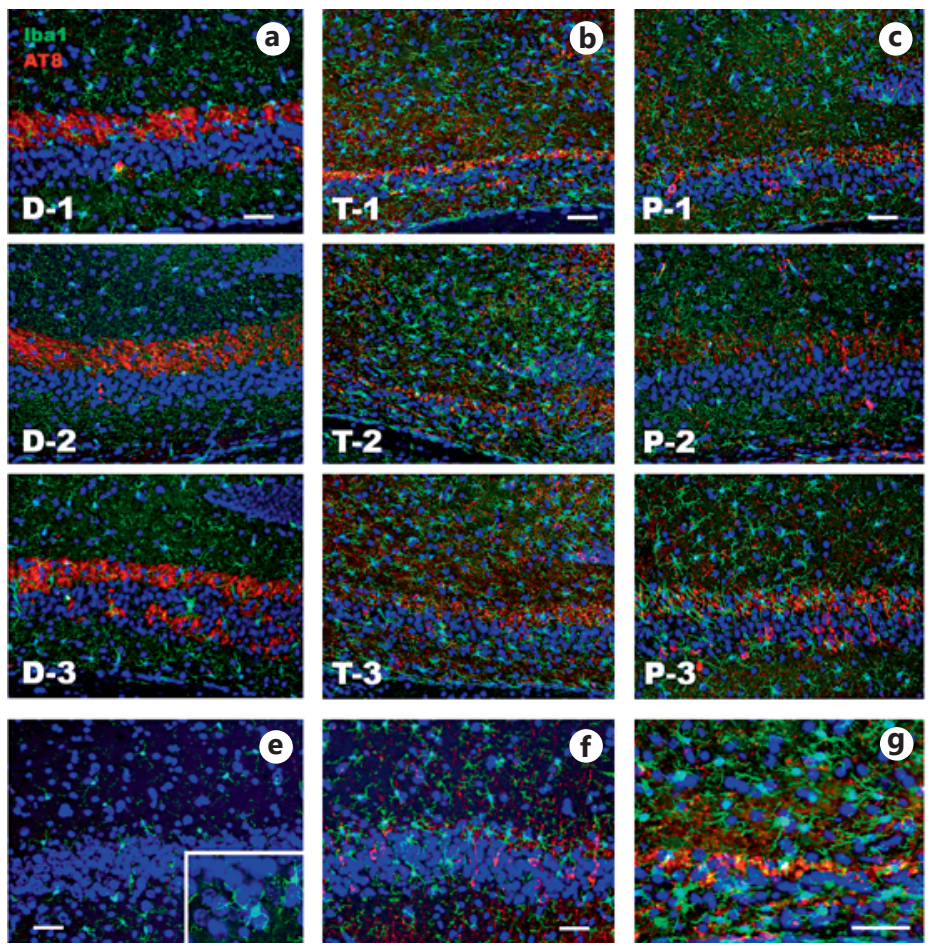
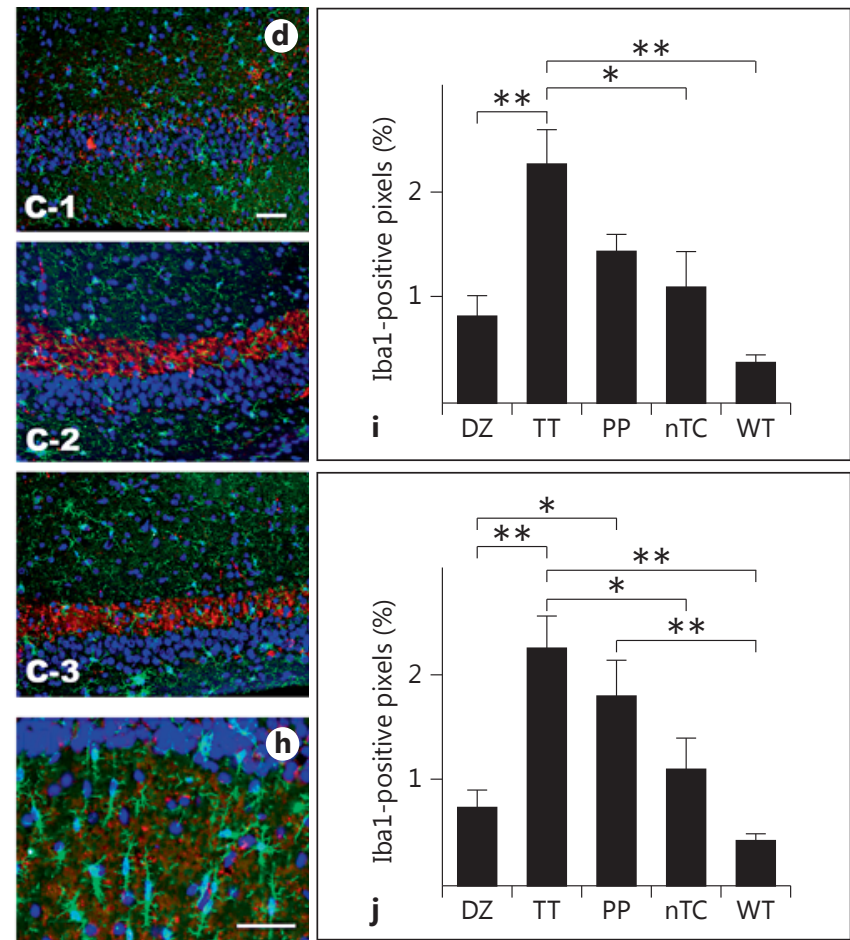

Fig. 2. Regulation of microglial activation by anticholinergic or cholinergic activity. The CA3 region of 10-month-old PS19 mice treated with DZ (D1-D3), TP (T1-T3) or PP (P1-P3) for 8 months, nTC (C1-C3; f) and 10-month-old wild-type mice (e) are doublestained with antibodies specific to Ibal (green) and AT8 (red). Wild-type mice show weakly stained Iba1-positive microglia with very thin processes (e; higher magnification in inset), while nTC PS19 mice show microglial activation in the CA3 region, with some microglia having a larger cytoplasm and thicker processes (C1-C3). $\mathbf{g}$ A higher-magnification image of the CA3 region from
TP-treated PS19 mice shows activated microglia with enlarged cytoplasm and thick processes in the CA3 region. $\mathbf{h}$ Elongated rodlike cytoplasm microglia with thick processes parallel to the nerve fibers in the CA1 region are frequently observed in the CA1 region. Scale bars $=50 \mu \mathrm{m}$. $\mathbf{i}$, j The Iba1-positive area in the CA1 (i) or CA3 (j) region was calculated using a PC and ImageJ software (NIH). WT $=$ Wild type. Statistical analysis clearly demonstrates that microglial activation is regulated by anticholinergic activity (TT $>$ PP) or cholinergic activity (DZ). ${ }^{*} \mathrm{p}<0.05,{ }^{* *} \mathrm{p}<0.01$ (ANOVA with post hoc test). activated with TP and PP, but were unchanged with DZ $[16,17]$. Thus, we cannot simply conclude that the effects of anticholinergic activity act directly opposite to the effects induced by cholinergic activity.

\section{Anticholinergic Activities, Neuroinflammation and Neurodegeneration}

The results described above indicate that anticholinergic activity might also be involved in enhancing pathological mechanisms leading to neurodegeneration in tauopathies. A positive correlation between microgliosis, as well as both tau pathology and neuronal loss, in PS19 mice treated with DZ, TP, PP or nTC (fig. 1) provided a mechanism for how anticholinergic activity could modi- fy neurodegeneration. Our previous studies demonstrated that microgliosis preceded tau pathology and neuronal loss, and early administration of an immunosuppressant, FK506, attenuated those pathologies in PS19 mice. This suggests neuroinflammation, including microglial activation, might initiate and promote processes leading to formation of tau pathologies and neurodegeneration [18]. Thus, one possible mechanism to explain the deterioration of neurological pathologies, such as tau pathology and neuronal loss, is due to modulation of inflammatory reactions by chronic administration of anticholinergics. Interestingly, DZ suppressed not only microgliosis in the brain, but also interleukin- $1 \beta$ and cyclooxygenase- 2 expression in the spleen of PS19 mice with lipopolysaccharide-induced systemic inflammation, indicating suppressive effects of DZ on not only central, but also 
systemic inflammation [17]. A series of studies demonstrated that ACh has an anti-inflammatory effect, and there are some reports demonstrating AChEIs exert inhibitory influence on neuroinflammation and generalized systemic inflammation [27-30]. Peripheral administration of AChEIs, or an antisense oligonucleotide to AChE, significantly attenuated interleukin- $1 \beta$ production in the hippocampus and blood of mice, with concomitant reduced AChE activity [27]. In mice, AChEIs also suppressed tumor necrosis factor (TNF) production in the blood and spleen following lipopolysaccharide-induced systemic inflammation [28]. There are two types of ACh receptors (AChRs), muscarinic and nicotinic receptors, and $\alpha_{7}$-nicotinic $\mathrm{AChR}$, a nicotinic AChR, plays a major role in the anti-inflammatory response in peripheral macrophages and central microglia $[31,32]$. Interestingly, the suppressive effect on blood TNF was reversed by surgical transection of the cervical vagus nerve or pretreatment with atropine sulfate, a blood-brain barrierpermeable muscarinic receptor antagonist. However, atropine methyl nitrate, a blood-brain barrier-impermeable muscarinic receptor antagonist, produced no effect. Moreover, a highly selective, centrally acting AChEI, huperzine A, significantly reduced blood TNF levels during chronic inflammation in rats $[33,34]$. Central muscarinic cholinergic activation by stimulating a postsynaptic muscarinic AChR (M1) agonist or inhibiting a presynaptic muscarinic AChR (M2) suppressed systemic TNF release in endotoxemic rats. Interestingly, blocking the peripheral muscarinic receptor did not abolish anti-inflammatory signaling, indicating that peripheral muscarinic receptor stimulation is not required for the cholinergic anti-inflammatory pathway, but central muscarinic receptor simulation exhibits an anti-inflammatory reaction [35]. Moreover, galantamine, an AChEI, significantly reduced serum TNF in endotoxemic mice, while administration of a centrally acting muscarinic receptor antagonist abolished the suppression of TNF by galantamine. Thus, the central anticholinergic (antimuscarinic) activity probably enhances neuroinflammation and systemic inflammation by blocking central muscarinic AChR [36]. In addition, chronic nicotine administration to a $3 \times \mathrm{Tg}$ AD mouse model (APPswe, P301L tau and PS1M146V) demonstrated significant age-dependent decreases in steady-state levels of $\alpha_{7}$-nicotinic AChR and exacerbation of tau pathology, but did now show any $A \beta$ pathological changes, indicating selective adverse effects of nicotine on tau [37]. Meanwhile, a recent study using a selective $\alpha_{7^{-}}$ nicotinic AChR agonist, A-582941, demonstrated enhanced cognition without alternation in pathological changes including $A \beta$ deposit, tau phosphorylation and inflammation [38]. In either case, stimulation of a nicotinic AChR might show no beneficial effect on tau pathology. Therefore, in PS19 mice, a central muscarine-dependent mechanism (anti-inflammatory mechanism) might play a more crucial role than a nicotine-dependent mechanism in suppressing tau pathology and neurodegeneration, as well as in reducing inflammatory responses in central and peripheral organs when $\mathrm{DZ}$ is administered.

It is well known that cholinergic function is profoundly depleted in $\mathrm{AD}$ brains. $\mathrm{A} \beta$ peptides suppress various steps of ACh synthesis and are released at low concentrations in cell culture and hippocampal slice studies, even at concentrations below those showing neurotoxic effects [39]. Direct injection of $A \beta$ into the murine brain demonstrated that $A \beta$ results in reduced $A C h$ production, cholinergic neurotoxicity, impaired learning and memory performance and inflammatory reactions characterized by interleukin- $1 \beta$ production and microglial activation [40-45]. Studies using AD model mice that develop agedependent senile plaques have shown $\mathrm{A} \beta$-dependent disruption of hippocampal ACh release, neuronal damage and axonal loss accompanied by impaired habituation learning [46-51]. Some studies have shown cholinergic nerve terminal degenerative pathology prior to deposition of $\mathrm{A} \beta$-containing neurotic plaques, as well as cholinergic nerve terminal degeneration and noncholinergic nerve sprouting surrounding plaques $[47,50]$. Interestingly, a report using an $\mathrm{AD}$ mouse model showed that anti- $\mathrm{A} \beta$ antibody treatment restores hippocampal ACh release and high-affinity choline uptake, indicating $A \beta$ directly interrupts ACh release in vivo [48]. This indicates A $\beta$ itself acts like anticholinergics. Meanwhile, a recent study reported rapid $A \beta$ deposition and cognitive impairment after cholinergic denervation induced by injections of a cholinergic specific immunotoxin, p75-SAP, into the nucleus basalis of Meynert and medial septum in APP/PS1 mice. Real-time monitoring by in vivo multiphoton microscopy demonstrated a significant increase in the deposition of senile plaques as soon as 7 days after cholinergic denervation, and biochemical analysis revealed enhanced tau phosphorylation [52]. These results demonstrated that $\mathrm{A} \beta$ not only preferentially affected functional cholinergic terminations prior to senile plaque formation, but also that cholinergic denervation enhanced $\mathrm{AD}$ pathologies. $\mathrm{A} \beta$ induces anticholinergic activity, and anticholinergic activity might enhance $A D$ pathologies including $A \beta$, tau and neuroinflammation (and vice versa).

It is well known that neuroinflammation plays an important part in the pathomechanism of neurodegenera- 
tion in $\mathrm{AD}$ and other neurodegenerative disorders [53]. Microglia and astroglia are known to be activated in the $\mathrm{AD}$ brain, and these reactive glia not only associate with plaques, but also parallel tangles in $\mathrm{AD}$ with upregulated expression of a variety of inflammatory cytokines and proteins including interleukin- $1 \beta$, TNF- $\alpha$, transforming growth factor- $\beta$ and $C$-reactive protein $[54,55]$. Recent studies about modulation of inflammation in transgenic models of $\mathrm{AD}$ provided evidence that enhanced inflammation is linked with increases in $A \beta$ generation, $A \beta$ aggregation and tau phosphorylation [56].

\section{Conclusion}

Neuropathological and biochemical studies have clearly demonstrated severe cholinergic dysfunction in $\mathrm{AD}$ and shown that anticholinergic activity leads to a de- cline of cognitive function and increases the risk of dementia. However, it is still unclear whether anticholinergic activity enhances $\mathrm{AD}$-related pathology and neurodegeneration; sufficient research has not yet been done in humans to support this conclusion. Taking into account the studies showing the central muscarinic cholinergic system regulates systemic and central inflammation, and the fact that inflammation plays a part in the pathogenesis of $\mathrm{AD}$ and $\mathrm{AD}$-related disorders, anticholinergic activity might promote the pathological process, and might initiate and/or enhance neuropathological changes in $\mathrm{AD}$ and AD-related disorders.

\section{Acknowledgment}

This work was partially supported by funding form Tsuchiya Zaidan to Y.Y. We thank Eisai (Japan) for providing DZ.

\section{References}

1 Arendt T, Bigl V, Arendt A, Tennstedt A: Loss of neurons in the nucleus basalis of Meynert in Alzheimer's disease, paralysis agitans and Korsakoff's disease. Acta Neuropathol 1983; 61:101-108

-2 Francis PT, Palmer AM, Snape M, Wilcock GK: The cholinergic hypothesis of Alzheimer's disease: a review of progress. J Neurol Neurosurg Psychiatry 1999;66:137-147.

3 Farlow MR: Do cholinesterase inhibitors slow progression of Alzheimer's disease? Int J Clin Pract Suppl 2002;127:37-44.

4 Giacobini E: Do cholinesterase inhibitors have disease-modifying effects in Alzheimer's disease? CNS Drugs 2001;15:85-91.

5 Giacobini E: Is anti-cholinesterase therapy of Alzheimer's disease delaying progression? Aging (Milano) 2001;13:247-254.

-6 Hashimoto M, Kazui H, Matsumoto K, Nakano Y, Yasuda M, et al: Does donepezil treatment slow the progression of hippocampal atrophy in patients with Alzheimer's disease? Am J Psychiatry 2005;162:676-682.

7 Wagg A: The cognitive burden of anticholinergics in the elderly - implications for the treatment of overactive bladder. Eur Urol Rev 2012;7:42-49.

8 Ancelin ML, Artero S, Portet F, Dupuy AM, Touchon J, et al: Non-degenerative mild cognitive impairment in elderly people and use of anticholinergic drugs: longitudinal cohort study. BMJ 2006;332:455-459.

-9 Carriere I, Fourrier-Reglat A, Dartigues JF, Rouaud O, Pasquier F, et al: Drugs with anticholinergic properties, cognitive decline, and dementia in an elderly general population: the 3-City Study. Arch Intern Med 2009;169: 1317-1324.
10 Moore AR, O'Keeffe ST: Drug-induced cognitive impairment in the elderly. Drugs Aging 1999;15:15-28.

11 Tune L, Carr S, Hoag E, Cooper T: Anticholinergic effects of drugs commonly prescribed for the elderly: potential means for assessing risk of delirium. Am J Psychiatry 1992;149: 1393-1394.

12 Mulsant BH, Pollock BG, Kirshner M, Shen C, Dodge $\mathrm{H}$, et al: Serum anticholinergic activity in a community-based sample of older adults: relationship with cognitive performance. Arch Gen Psychiatry 2003;60:198-203.

$\checkmark 13$ Hori K, Konishi K, Watanabe K, Uchida H, Tsuboi T, et al: Influence of anticholinergic activity in serum on clinical symptoms of Alzheimer's disease. Neuropsychobiology 2011;63:147-153.

14 Fox C, Smith T, Maidment I, Chan WY, Bua $\mathrm{N}$, et al: Effect of medications with anti-cholinergic properties on cognitive function, delirium, physical function and mortality: a systematic review. Age Ageing 2014;43:604-615.

15 Perry EK, Kilford L, Lees AJ, Burn DJ, Perry RH: Increased Alzheimer pathology in Parkinson's disease related to antimuscarinic drugs. Ann Neurol 2003;54:235-238.

16 Yoshiyama Y, Kojima A, Itoh K, Uchiyama T, Arai K: Anticholinergics boost the pathological process of neurodegeneration with increased inflammation in a tauopathy mouse model. Neurobiol Dis 2012;45:329-336.

17 Yoshiyama Y, Kojima A, Ishikawa C, Arai K: Anti-inflammatory action of donepezil ameliorates tau pathology, synaptic loss, and neurodegeneration in a tauopathy mouse model. J Alzheimers Dis 2010;22:295-306.
18 Yoshiyama Y, Higuchi M, Zhang B, Huang SM, Iwata N, et al: Synapse loss and microglial activation precede tangles in a P301S tauopathy mouse model. Neuron 2007;53: 337-351.

19 Jack CR Jr, Wiste HJ, Knopman DS, Vemuri P, Mielke MM, et al: Rates of beta-amyloid accumulation are independent of hippocampal neurodegeneration. Neurology 2014; 82: 1605-1612.

20 Yoshiyama Y, Lee VM, Trojanowski JQ: Therapeutic strategies for tau mediated neurodegeneration. J Neurol Neurosurg Psychiatry 2013;84:784-795.

21 Clavaguera F, Bolmont T, Crowther RA, Abramowski D, Frank S, et al: Transmission and spreading of tauopathy in transgenic mouse brain. Nat Cell Biol 2009;11:909-913.

22 Kobayashi F, Yageta Y, Yamazaki T, Wakabayashi E, Inoue M, et al: Pharmacological effects of imidafenacin (KRP-197/ONO-8025), a new bladder selective anti-cholinergic agent, in rats. Comparison of effects on urinary bladder capacity and contraction, salivary secretion and performance in the Morris water maze task. Arzneimittelforschung 2007;57:147-154.

23 Oka T, Nakano K, Kirimoto T, Matsuura N: Effects of antimuscarinic drugs on both urinary frequency and cognitive impairment in conscious, nonrestrained rats. Jpn J Pharmacol 2001;87:27-33.

24 Suzuki M, Noguchi Y, Okutsu H, Ohtake A, Sasamata M: Effect of antimuscarinic drugs used for overactive bladder on learning in a rat passive avoidance response test. Eur J Pharmacol 2007;557:154-158. 
-25 Ishihara T, Hong M, Zhang B, Nakagawa Y, Lee MK, et al: Age-dependent emergence and progression of a tauopathy in transgenic mice overexpressing the shortest human tau isoform. Neuron 1999;24:751-762.

26 Sahara N, Murayama M, Lee B, Park JM, Lagalwar S, et al: Active c-jun N-terminal kinase induces caspase cleavage of tau and additional phosphorylation by GSK-3beta is required for tau aggregation. Eur J Neurosci 2008;27:2897-2906.

-27 Pollak Y, Gilboa A, Ben-Menachem O, BenHur T, Soreq H, et al: Acetylcholinesterase inhibitors reduce brain and blood interleukin1beta production. Ann Neurol 2005;57:741745 .

-28 Tyagi E, Agrawal R, Nath C, Shukla R: Effect of anti-dementia drugs on LPS induced neuroinflammation in mice. Life Sci 2007;80: 1977-1983.

-29 Satapathy SK, Ochani M, Dancho M, Hudson LK, Rosas-Ballina M, et al: Galantamine alleviates inflammation and other obesity-associated complications in high-fat diet-fed mice. Mol Med 2011;17:599-606.

-30 Jiang Y, Zou Y, Chen S, Zhu C, Wu A, et al: The anti-inflammatory effect of donepezil on experimental autoimmune encephalomyelitis in C57 BL/6 mice. Neuropharmacology 2013; 73:415-424.

-31 Thomsen MS, Mikkelsen JD: The alpha7 nicotinic acetylcholine receptor ligands methyllycaconitine, NS6740 and GTS-21 reduce lipopolysaccharide-induced TNF-alpha release from microglia. J Neuroimmunol 2012;251: 65-72.

-32 Pavlov VA, Tracey KJ: Controlling inflammation: the cholinergic anti-inflammatory pathway. Biochem Soc Trans 2006;34:1037-1040.

33 Kolgazi M, Uslu U, Yuksel M, VeliogluOgunc A, Ercan F, et al: The role of cholinergic anti-inflammatory pathway in acetic acidinduced colonic inflammation in the rat. Chem Biol Interact 2013;205:72-80.

- 34 Wang J, Zhang HY, Tang XC: Huperzine A improves chronic inflammation and cognitive decline in rats with cerebral hypoperfusion. J Neurosci Res 2010;88:807-815.

35 Pavlov VA, Ochani M, Gallowitsch-Puerta M, Ochani K, Huston JM, et al: Central muscarinic cholinergic regulation of the systemic inflammatory response during endotoxemia. Proc Natl Acad Sci USA 2006;103:5219-5223.
36 Pavlov VA, Parrish WR, Rosas-Ballina M, Ochani M, Puerta M, et al: Brain acetylcholinesterase activity controls systemic cytokine levels through the cholinergic anti-inflammatory pathway. Brain Behav Immun 2009;23: $41-45$.

37 Oddo S, Caccamo A, Green KN, Liang K, Tran L, et al: Chronic nicotine administration exacerbates tau pathology in a transgenic model of Alzheimer's disease. Proc Natl Acad Sci USA 2005; 102:3046-3051.

38 Medeiros R, Castello NA, Cheng D, Kitazawa $\mathrm{M}$, Baglietto-Vargas D, et al: Alpha7 nicotinic receptor agonist enhances cognition in aged $3 \mathrm{xTg}-\mathrm{AD}$ mice with robust plaques and tangles. Am J Pathol 2014;184:520-529.

39 Kar S, Slowikowski SP, Westaway D, Mount HT: Interactions between beta-amyloid and central cholinergic neurons: implications for Alzheimer's disease. J Psychiatry Neurosci 2004;29:427-441.

40 Abe E, Casamenti F, Giovannelli L, Scali C, Pepeu G: Administration of amyloid betapeptides into the medial septum of rats decreases acetylcholine release from hippocampus in vivo. Brain Res 1994;636:162-164.

41 Blusztajn JK, Berse B: The cholinergic neuronal phenotype in Alzheimer's disease. Metab Brain Dis 2000;15:45-64.

42 Harkany T, De Jong GI, Soos K, Penke B, Luiten PG, et al: Beta-amyloid (1-42) affects cholinergic but not parvalbumin-containing neurons in the septal complex of the rat. Brain Res 1995;698:270-274.

43 Itoh A, Nitta A, Nadai M, Nishimura K, Hirose $\mathrm{M}$, et al: Dysfunction of cholinergic and dopaminergic neuronal systems in beta-amyloid protein-infused rats. J Neurochem 1996; 66:1113-1117.

44 Giovannelli L, Casamenti F, Scali C, Bartolini L, Pepeu G: Differential effects of amyloid peptides beta-(1-40) and beta-(25-35) injections into the rat nucleus basalis. Neuroscience 1995;66:781-792.

45 Watanabe T, Iwasaki K, Ishikane S, Naitou T, Yoshimitsu Y, et al: Spatial memory impairment without apoptosis induced by the combination of beta-amyloid oligomers and cerebral ischemia is related to decreased acetylcholine release in rats. J Pharmacol Sci 2008; 106:84-91.
46 Bellucci A, Luccarini I, Scali C, Prosperi C, Giovannini MG, et al: Cholinergic dysfunction, neuronal damage and axonal loss in TgCRND8 mice. Neurobiol Dis 2006;23:260272.

$47 \mathrm{Hu} \mathrm{L}$ : The impact of A $\beta$-plaques on cortical cholinergic and non-cholinergic presynaptic boutons in Alzheimer's disease-like transgenic mice. Neuroscience 2003;121:421-432.

48 Bales KR: Cholinergic dysfunction in a mouse model of Alzheimer disease is reversed by an anti-A antibody. J Clin Invest 2006;116:825832

49 Wong TP, Debeir T, Duff K, Cuello AC: Reorganization of cholinergic terminals in the cerebral cortex and hippocampus in transgenic mice carrying mutated presenilin- 1 and amyloid precursor protein transgenes. J Neurosci 1999;19:2706-2716.

50 German DC, Yazdani U, Speciale SG, Pasbakhsh P, Games D, et al: Cholinergic neuropathology in a mouse model of Alzheimer's disease. J Comp Neurol 2003;462:371-381.

-51 Perez SE, Dar S, Ikonomovic MD, De Kosky ST, Mufson EJ: Cholinergic forebrain degeneration in the APPswe/PS1DeltaE9 transgenic mouse. Neurobiol Dis 2007;28:3-15.

52 Ramos-Rodriguez JJ, Pacheco-Herrero M, Thyssen D, Murillo-Carretero MI, Berrocoso E, et al: Rapid beta-amyloid deposition and cognitive impairment after cholinergic denervation in APP/PS1 mice. J Neuropathol Exp Neurol 2013;72:272-285.

-53 Akiyama H, Barger S, Barnum S, Bradt B, Bauer J, et al: Inflammation and Alzheimer's disease. Neurobiol Aging 2000;21:383-421.

54 Serrano-Pozo A, Muzikansky A, Gomez-Isla T, Growdon JH, Betensky RA, et al: Differential relationships of reactive astrocytes and microglia to fibrillar amyloid deposits in $\mathrm{Alz}$ heimer disease. J Neuropathol Exp Neurol 2013;72:462-471.

55 Serrano-Pozo A, Mielke ML, Gomez-Isla T, Betensky RA, Growdon JH, et al: Reactive glia not only associates with plaques but also parallels tangles in Alzheimer's disease. Am J Pathol 2011;179:1373-1384.

56 Birch AM, Katsouri L, Sastre M: Modulation of inflammation in transgenic models of Alzheimer's disease. J Neuroinflamm 2014;11:25. 